\title{
Phase Separation Driven by External Fluctuations
}

\author{
J. García-Ojalvo \\ Departament de Física i Enginyeria Nuclear, Universitat Politècnica de Catalunya, \\ Colom 11, E-08222 Terrassa, Spain
}

A.M. Lacasta

Departament de Fúsica Aplicada, Universitat Politècnica de Catalunya, Av. Dr. Gregorio Marañón, 50, E-08028 Barcelona, Spain

J.M. Sancho

Institute for Nonlinear Science, Department 0407, University of California, San Diego, 9500 Gilman Drive, La Jolla, California 92093-0407 and Departament d'Estructura i Constituents de la Matèria, Universitat de Barcelona, Av. Diagonal 647, E-08028 Barcelona, Spain

R. Toral

Departament de Física, Universitat de les Illes Balears and Instituto Mediterráneo de Estudios Avanzados (UIB-CSIC), E-07071 Palma de Mallorca, Spain

\begin{abstract}
The influence of external fluctuations in phase separation processes is analysed. These fluctuations arise from random variations of an external control parameter. A linear stability analysis of the homogeneous state shows that phase separation dynamics can be induced by external noise. The spatial structure of the noise is found to have a relevant role in this phenomenon. Numerical simulations confirm these results. A comparison with order-disorder noise induced phase transitions is also made.
\end{abstract}

PACS numbers: $05.40+\mathrm{j}, 47.20 . \mathrm{Ky}, 47.20 . \mathrm{Hw}$

The role of noise as an ordering agent has been broadly studied in recent years in the context of both temporal and spatiotemporal dynamics. In the temporal case, which was the one to be addressed earlier, external fluctuations were found to produce and control transitions (known as noise-induced transitions) from monostable to bistable stationary distributions in a large variety of physical, chemical and biological systems [1]. Spatiotemporal systems have been faced much more recently. In these cases, the combined effects of the spatial coupling and the noise terms acting upon the system variables may produce an ergodicity breaking of a bistable state, leading to phase transitions between spatially homogeneous and inhomogeneous phases. Results obtained in this field include critical-point shifts in standard models of phase transitions 22 4], pure noise-induced phase transitions [5], stabilization of propagating fronts [8], and noisedriven structures in pattern-formation processes [9 12 . In all these cases, the qualitative (and somewhat counterintuitive) effect of noise is to enlarge the domain of existence of the ordered phase in parameter space. It is the purpose of this Letter to analyse the role of fluctuations in a radically different type of spatiotemporal process, namely in phase separation dynamics. It will be shown that external noise can induce the phase separation process and that this effect is determined by the spatial correlation of the noise terms. An important conclusion is that phase separation does not occur, necessarily, for the same range of parameters for which the system presents a phase ordering process.

The dynamics of a large class of spatially-extended systems can be described in a general way by the following standard model [13]:

$$
\frac{\partial \psi(\vec{x}, t)}{\partial t}=-\mathcal{L}(\nabla)\left(\frac{\delta \mathcal{F}}{\delta \psi}\right)+\xi(\vec{x}, t)
$$

where $\psi(\vec{x}, t)$ is a dynamical field that describes the state of the system, $\mathcal{F}$ is a free energy functional, and $\xi(\vec{x}, t)$ is a space-dependent stochastic process that accounts for thermal fluctuations. Thermal equilibrium at temperature $T$ is reached at long times if the fluctuationdissipation relation holds:

$$
\left\langle\xi(\vec{x}, t) \xi\left(\vec{x}^{\prime}, t^{\prime}\right)\right\rangle=2 \varepsilon \mathcal{L}(\nabla) \delta\left(t-t^{\prime}\right) \delta\left(\vec{x}-\vec{x}^{\prime}\right)
$$

with $\varepsilon=k_{B} T$. The system is usually characterised by the behavior of the spatial average of the field $\psi$, which plays the role of an order parameter. The operator $\mathcal{L}(\nabla)$ does not alter the equilibrium state of the system $\left(\sim \exp \left(-\mathcal{F} / k_{B} T\right)\right)$, but only its transient dynamics. Two forms of $\mathcal{L}$ are usually adopted: for $\mathcal{L}=1$ (the so-called model $A$ ) the system evolves towards its equilibrium state without any constraint on the value of the order parameter; for $\mathcal{L}(\nabla)=-\nabla^{2}($ model $B)$ the order 
parameter is conserved throughout the dynamical evolution. Model $A$ is a prototype of ferromagnetic ordering, and model $B$ of phase separation dynamics following a quench from a high temperature homogeneous phase to a low temperature state. In this latter case, according to the value of the order parameter and the quench location, the evolution might proceed either by spinodal decomposition or by nucleation [14.

Far from the critical point, the dynamics is not qualitatively affected by the presence of the thermal noise term $\xi(\vec{x}, t)$. Instead, we will consider the situation in which there is an additional source of noise, $\eta(\vec{x}, t)$. This happens, for instance, when one of the externally controlled system parameters is subjected to fluctuations. As these fluctuations are external, they do not usually verify the fluctuation-dissipation relation (2) and the system is no longer at equilibrium. We will show that, in the case of model $B$, these external fluctuations can induce a phase separation in the system. Although this is a reminiscence of noise-induced phase transitions reported earlier for model $A$ [2, 4. 15] (and, in what follows, we will make a comparison between the effect of external noise in both models), an unexpected and notorious feature is that the nature of the destabilizing terms due to the noise is intrinsically different for model $A$ and model $B$. We conclude that phase separation does not necessarily occur in the conserved order-parameter model $B$ at the same values for which model $A$ shows a noise-induced phase transition. It follows that, in contrast with what happens at equilibrium, both models have now different stationary distributions. Furthermore, a relevant result of our analysis is that the spatial structure of the noise plays an important and distinct role for both models. We assume the following correlation for the external noise with a characteristic correlation length $\lambda$ :

$$
\left\langle\eta(\vec{x}, t) \eta\left(\vec{x}^{\prime}, t^{\prime}\right)\right\rangle=2 \sigma^{2} \delta\left(t-t^{\prime}\right) g\left(\left|\vec{x}-\vec{x}^{\prime}\right| / \lambda\right)
$$

where $g$ is a (short-ranged) spatial correlation function. It is expected, and will be confirmed in what follows, that since model $B$ represents a domain-growth process, this correlation length will play a role far beyond the intuitive intensity-reduction effect of space-time noise correlation [3]. Correlation time of the noise should not have a parallel influence, since all time scales of the system are larger than those of the noise. Therefore, it seems that a finite (non-zero) correlation time of the noise would not differentiate between models $A$ and $B$, and will not be considered here (the time dependence of the correlation of $\eta(\vec{x}, t)$ will be assumed to be a Dirac delta, as shown in Eq.(3)).

Although our results are quite general, we shall work, for the sake of clarity, with the well known GinzburgLandau free energy

$$
\mathcal{F}=\int d \vec{x}\left[\frac{r}{2} \psi^{2}+\frac{1}{4} \psi^{4}+\frac{K}{2}|\vec{\nabla} \psi|^{2}\right]
$$

In the absence of additive (thermal) noise, phase separation occurs for $r<r_{c}=0$. If additive noise is present, the transition occurs at $r_{c}<0$. We will consider $r>0$, so that order will not appear spontaneously. The control parameter $r$ will be assumed to be subjected to external fluctuations, i.e. $r \rightarrow r+\eta(\vec{x}, t)$. The spatial correlation function $g$ is chosen to be a Gaussian of width $\lambda$ :

$$
g\left(\frac{\left|\vec{x}-\vec{x}^{\prime}\right|}{\lambda}\right)=\frac{1}{(\lambda \sqrt{2 \pi})^{d}} \exp \left(-\frac{\left|\vec{x}-\vec{x}^{\prime}\right|^{2}}{2 \lambda^{2}}\right),
$$

( $d$ is the $\vec{x}$-space dimension) which becomes a delta function in the limit $\lambda \rightarrow 0$. It is simpler to analyze the role of noise by using a lattice discretization in which the space vectors $\vec{x}$ take values $x_{i}(i=1, \ldots, N)$, defined on regular lattice of linear cell size $\Delta x=1$. The field $\psi(\vec{x}, t)$ then becomes a discrete set of variables $\psi_{i}(t)$ and similar notation is used for the random fields $\eta_{i}(t)$ and $\xi_{i}(t)$. Under these considerations, the lattice version of model (1) with the Ginzburg-Landau free energy (4) is:

$$
\dot{\psi}_{i}=-\mathcal{L}_{L}\left(r \psi_{i}+\eta_{i} \psi_{i}+\psi_{i}^{3}-K \nabla_{L}^{2} \psi_{i}\right)+\xi_{i},
$$

where $\mathcal{L}_{L}=1$ for model $A$ and $\mathcal{L}_{L}=-\nabla_{L}^{2}$ for model $B$. $\nabla_{L}^{2}$ is the lattice Laplacian operator. Finally, in this version, the external noise has a correlation function $g_{|i-j|}$ which is the discrete inverse Fourier transform of $\hat{g}_{k}$, the corresponding lattice version of the Fourier transform of (5), namely (in two dimensions):

$$
\hat{g}_{k}=\exp \left(-\frac{\lambda^{2}}{2}\left(\sin \left(k_{x} / 2\right)^{2}+\sin \left(k_{y} / 2\right)^{2}\right)\right)
$$

There is no closed analytical form for $g_{|i-j|}$ and the desired values $g_{0}$ and $g_{1}$ (see later) must be obtained numerically.

The transition towards an ordered state can be analyzed by studying the stability of the homogeneous phase $\psi_{i}=0$. The early time evolution of the statistical moments of $\psi_{i}$ in Fourier space can be obtained in a linear approximation. For example, the second moment (structure function) is defined as $S_{k}(t)=\frac{1}{N}\left\langle\hat{\psi}_{k} \hat{\psi}_{-k}\right\rangle$, where $N$ is the number of points of the system. Making use of the Stratonovich calculus and Novikov's theorem, its evolution equation is [1]:

$$
\frac{d S_{k}(t)}{d t}=-2 \omega(k) S_{k}(t)+\frac{1}{N} f(k) \sum_{k} \hat{g}_{k} S_{k}(t)+2 \varepsilon
$$

Hence the second moment equation contains a term which globally couples Fourier modes and a constant term due to thermal noise. The particular values of the dispersion relation $\omega(k)$ and of the mode-coupling coefficient $f(k)$ differ for models $A$ and $B$. For model $A$, the result is well known [4]

$$
\omega^{A}(k)=r_{e f f}^{A}+K k^{2}, \quad f^{A}(k)=1
$$


with an effective control parameter $r_{e f f}^{A}=r-\sigma^{2} g_{0}$. For model $B$ the situation is drastically different:

$$
\omega^{B}(k)=r_{e f f}^{B} k^{2}+K_{e f f}^{B} k^{4}, \quad f^{B}(k)=k^{2}
$$

with effective control parameter $r_{\text {eff }}^{B}=r+\sigma^{2} \nabla_{L}^{2} g_{0}$ and effective diffusion coefficient $K_{\text {eff }}^{B}=K-\sigma^{2} g_{1}$. Two main differences are observed with respect to model $A$ : the diffusion coefficient $K$ is also renormalized by the correlated external noise, and the noise-induced shift of the control parameter $r$ depends now, through the Laplace operator $\nabla_{L}^{2}$, on the spatial structure of the noise correlation, i.e. not only on the same-site correlation $g_{0}$, but also on the nearest-neighbor correlation $g_{1}$. These differences will reveal itself in the position of the transition point where the homogeneous state loses stability and phase separation appears.

When neglecting the mode-coupling terms in Eq.(8), it is readily seen that perturbations grow when $w(k)^{A, B}<0$ for some interval of $k$ values. We have checked, by means of a numerical integration of Eq. (8), that modecoupling terms hardly influence the position of the transition curves. Hence the transition point is characterised by $r_{\text {eff }}=0$ for both model $A$ and model $B$. The critical value of the control parameter $r$ and its dependence on the spatial structure of the noise is, however, different in the two cases:

$$
\begin{array}{ll}
\operatorname{model} A: & r_{c}=\sigma^{2} g_{0} \\
\operatorname{model} B: & r_{c}=-\sigma^{2} \nabla_{L}^{2} g_{0}=\sigma^{2} 2 d\left(g_{0}-g_{1}\right)
\end{array}
$$

Figure 1 shows the transition curves between homogenous and inhomogeneous states in the $\left(\lambda, \sigma^{2}\right)$ plane for models $A$ and $B$ for a fixed value, $r=0.2$, of the control parameter and spatial dimension $d=2$. All points located above the curves shown in this phase diagram are in an inhomogeneous state, which corresponds to an ordered phase in model $A$ (solid curve) and to phase separation in model $B$ (dashed curve). The $\lambda$-dependence of the model- $A$ curve is merely due to the natural "softening" effect of noise correlation [3,11. In the case of model $B$, on the other hand, additional, non-trivial dependence on the correlation length is introduced via the Laplace operator. As a consequence, for small $\lambda$, the transition in model $B$ occurs sooner in model $B$ than in model $A$, whereas the situation is the opposite for large values of $\lambda$. A crossing of the two transition curves occurs for an intermediate value of $\lambda \approx 1.8$. We stress again that the presence of ordered regions in the phase diagram of figure 1 is due to the presence of a multiplicative noise on the model, since we are taking $r=0.2$ which is larger than the mean-field critical value $r_{c}=0$.

The lines drawn in the phase diagram of Fig. 1 have been obtained in a linear approximation (11). It is presumable that this linear stability analysis will provide the position of the transition points up to leading order of approximation 涵. In order to corroborate the results obtained by means of the linear stability analysis, equations (6) have been integrated numerically for models $A$ and $B$ in dimension $d=2$. A standard stochastic algorithm is used in order to handle both the additive and multiplicative noise terms [16]. Gaussianly-distributed random vectors are generated by means of a numerical inversion method, optimised to efficiently produce large quantities of Gaussian random numbers [17]. According to the previous discussion, the spatially correlated external noise is generated in Fourier space with the desired correlation function (7), and transformed back to real space at each integration time step 11 .

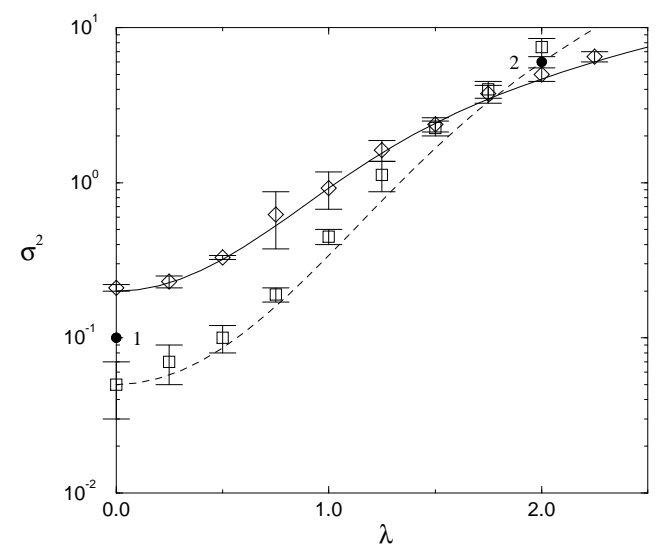

FIG. 1. Phase diagram in the $\left(\lambda, \sigma^{2}\right)$ plane for model A (solid line) and model B (dashed line), obtained from a linear stability analysis (8). Points correspond to numerical simulations of the full model (6), (diamonds: model A, squares: model B) $\left(r=0.2, K=1\right.$, and $\left.\varepsilon=10^{-4}\right)$.

A unified criterion for the existence of an ordered phase in model $A$ and of phase separation in model $B$ is the growth of the averaged second moment of $\psi$ in real space (the averaged first moment is not useful for model $B$ because this model conserves the order parameter). We define this quantity as $J(t)=\frac{1}{N}\left\langle\sum_{i} \psi_{i}^{2}(t)\right\rangle$ or, alternatively, as $J(t)=\sum_{k} S_{k}(t)$. The instability point is thus defined so that, below it, $J(t)$ decays to a thermal-noise background at large times and, above it, it grows to a non-zero steady-state value $J_{s t}$. In this way, one can determine numerically the phase diagram of the system. The numerical results are represented in Fig. 1 as diamonds (model $A$ ) and squares (model $B)$. It can be observed that the simulations of the full nonlinear models reasonably adjust to the predictions of the linear analysis. The agreement starts to fail at high values of $\lambda$ and $\sigma^{2}$. In fact, according to previous observations in model $A$ [15] and other models [5.6.6, the transition curves might be expected to exhibit, for higher values of the noise intensity, a reentrant branch towards the $\lambda=0$ axis. These reentrant transitions are hard to observe numerically be- 
cause of the large noise intensities involved.

Fig. 2 shows two patterns of a system evolving according to model $B$, for point 1 in the phase diagram of Fig. 1. Depending on the initial conditions we get spinodal decomposition (Fig. 2a, with $\langle\psi(\vec{x}, 0)\rangle=0$ ), or nucleation (Fig. 2b, with $\langle\psi(\vec{x}, 0)\rangle=0.1$ ). For the same values of the noise parameters a homogeneous phase is obtained for model $A$.

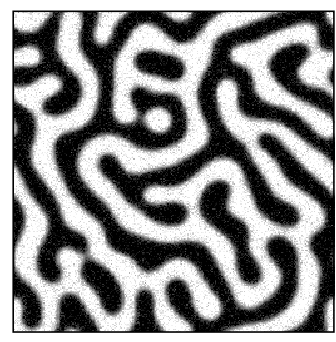

(a)

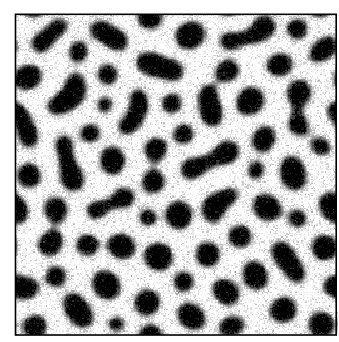

(b)
FIG. 2. Spatial patterns of model B: a) Spinodal decomposition and $\mathrm{b})$ Nucleation. $\left(t=2500, \lambda=0, \sigma^{2}=0.1\right.$ and $\left.\varepsilon=10^{-4}\right)$

For larger values of the noise parameters a reverse situation is found. Fig. 3 shows a spatial pattern of model $A$ for point 2 in the phase diagram of Fig. 1 . Now the homogeneous phase corresponds to model $B$.

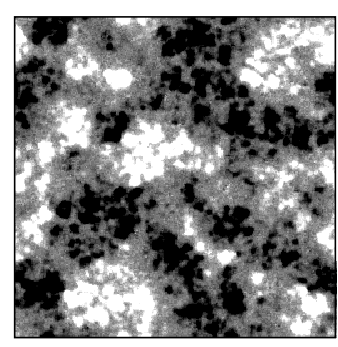

FIG. 3. Spatial pattern of model A. ( $t=100, \lambda=2$, $\sigma^{2}=6$ and $\varepsilon=10^{-4}$.)

In conclusion, we have demonstrated for the first time the ordering role of external noise in processes of phase separation. The study has concentrated on the conserved time-dependent Ginzburg-Landau model, although our results are not restricted to this particular model. External noise is found to enhance the phase separation process, and this effect is observed to be modified by spatial correlation of the noise, which increases the efficiency of fluctuations for small correlation lengths and decreases it for large correlation lengths. Future work on this issue should analyse the important effect of external noise on the dynamical scaling of the phase separation process. Additional theoretical analysis such as a mean-field approach [2,5,6] could be useful in determining whether a reentrant transition should be expected in this model.
We acknowledge financial support from the Dirección General de Investigación Científica y Técnica (Spain), under projects PB96-0421, PB94-1167 and PB94-1172), and computing support from Fundació Catalana per a la Recerca.

[1] W. Horsthemke and R. Lefever, Noise-Induced Transitions (Springer-Verlag, Berlin, 1984).

[2] C. Van den Broeck, J.M.R. Parrondo, J. Armero, and A. Hernández-Machado, Phys. Rev. E 49, 2639 (1994).

[3] J. García-Ojalvo and J.M. Sancho, Phys. Rev. E 49, 2769 (1994).

[4] A. Becker and L. Kramer, Phys. Rev. Lett. 73, 955 (1994); Physica D 90, 408 (1995).

[5] C. Van den Broeck, J.M.R. Parrondo, and R. Toral, Phys. Rev. Lett. 73, 3395 (1994).

[6] C. Van den Broeck, J.M.R. Parrondo, R. Toral, and R. Kawai, Phys. Rev. E 55, 4084 (1997).

[7] S.H. Park and S. Kim, Phys. Rev. E 53, 3425 (1996).

[8] J. Armero, J.M. Sancho, J. Casademunt, A.M. Lacasta, L. Ramírez-Piscina, and F. Sagués, Phys. Rev. Lett. 76, 3045 (1996).

[9] J. García-Ojalvo, A. Hernández-Machado, and J.M. Sancho, Phys. Rev. Lett. 71, 1542 (1993).

[10] J.M.R. Parrondo, C. Van den Broeck, J. Buceta, and F.J. de la Rubia, Physica A 224, 153 (1996).

[11] J. García-Ojalvo and J.M. Sancho, Phys. Rev. E 53, 5680 (1996).

[12] G.D. Lythe, Phys, Rev. E 53, R4271 (1996).

[13] P.C. Hohenberg and B.I. Halperin, Rev. Mod. Phys. 49,435 (1977).

[14] J.D. Gunton, M. San Miguel and P. Sahni, in Phase Transitions and Critical Phenomena, vol. 8, edited by C. Domb and J.L. Lebowitz, Academic, New York (1983).

[15] J. García-Ojalvo, J.M.R. Parrondo, J.M Sancho, and C. Van den Broeck, Phys. Rev. E 54, 6918 (1996).

[16] T.C. Gard, Introduction to Stochastic Differential Equations (Marcel Dekker, New York, 1987).

[17] R. Toral and A. Chakrabarti, Comp. Phys. Commun. 74, 327 (1993). 